\title{
Informe sobre a Cooperação Brasil-Canadá em Promoção da Saúde*
}

\author{
Report on the Cooperation Brazil-Canada \\ in Health Promotion
}

Lenira Zancan 1

Leila Adesse 1

\footnotetext{
1 Departamento de Ciências Sociais, Escola Nacional de Saúde Pública, Fiocruz. Av. Leopoldo Bulhões 1480, 2o andar, Manguinhos, 21041-210, Rio de Janeiro RJ. lenazan@ensp.fiocruz.br * Informe elaborado a partir do Projeto Ações Intersetoriais para a Saúde, parceria entre a ENSP/ Fiocruz/Abrasco/CPHA.
}

\begin{abstract}
This report presents the Project Development Phase of a Cooperation on Health Promotion involving the National School of Public Health (ENSP), the Brazilian Association of Collective Health (ABRASCO), and the Canadian Association of Public Health (CPHA). That experience evidenced that health promotion and local development are guided by common values and similar strategies for action, which are based on public participation, empowerment, and intersectoral collaboration. In Brazil the strategies for health promotion and local development are still undergoing and, for theirs complexity, need to be systematically analyzed. Within this context, the Canadian and Brazilian partners propose to assist selected municipalities across the country in undertaking a mix of health promotion interventions and capacity building activities on local development and intersectoral action for health. This collaborative agenda, based on theory-driven evaluative approaches, will contribute to build a model and tools for reflexive pratices on local development on health, and to create and sustain improvements in social and physical environments and to improved health outcomes.
\end{abstract}

Key words Local development, Health promotion, Intersectoral action, Evaluation
Resumo O trabalho relata a experiência de cooperação no campo da Promoção da Saúde, estabelecida entre a Escola Nacional de Saúde Pública (ENSP), a Associação Brasileira de Saúde Coletiva (Abrasco) e a Associação Canadense de Saúde Pública (CPHA) nos últimos quatro anos. Tal experiência tem evidenciado que a promoção da saúde e o desenvolvimento local são guiados por valores comuns e estratégias de ação similares, fundamentados em participação social, empowerment e colaboração intersetorial. No Brasil, as estratégias para promoção da saúde e desenvolvimento local ainda são insipientes e, por sua complexidade, necessitam ser sistematicamente analisadas. Nesse contexto, os parceiros canadenses e brasileiros se propõem, através de um novo Projeto de Cooperação, apoiar municípios selecionados nas várias regiões do país, na implementação e avaliação de intervenções em promoção da saúde e na construção de capacidades para o desenvolvimento local e ação intersetorial para a saúde. Essa agenda colaborativa toma por base as teorias da avaliação participativa, contribuindo para a construção de modelos e ferramentas de reflexive practices em desenvolvimento local e promoção da saúde, criando processos sustentáveis de melhoria dos ambientes físicos e sociais com impacto na saúde.

Palavras-chave Desenvolvimento local, Promoção da saúde, Ação intersetorial, Avaliação 
Nos últimos anos, a Associação Canadense de Saúde Pública (CPHA) colaborou com a Escola Nacional de Saúde Pública (ENSP/Fiocruz) e com a Associação Brasileira de Saúde Coletiva (Abrasco), com o objetivo de criar conhecimento e experiência por intermédio do Projeto de Promoção de Saúde em Ação (PPSA). A liderança do Canadá no campo da promoção da saúde é reconhecida mundialmente. Esta iniciativa buscava englobar a teoria, a pesquisa e o ensino em promoção da saúde, com a prática da saúde pública. O intercâmbio técnico fortaleceu e expandiu ensinamentos e ações de promoção da saúde já existentes na Escola Nacional de Saúde Pública da Fundação Oswaldo Cruz.

O PPSA, ao longo dos três anos e meio de vigência, constituiu-se em fator catalisador e estimulador de práticas anteriormente dispersas e fragmentadas, possibilitando o desenvolvimento de novos projetos e ações envolvendo a academia, o serviço e a comunidade. Evidências disso estão presentes nos novos programas e estratégias organizacionais da ENSP através de seu Centro de Saúde Escola, na incorporação de disciplinas e cursos na grade e na abertura de linha de investigação em promoção da saúde, reunindo diversos departamentos, pesquisadores e profissionais de saúde.

Com o apoio de consultores canadenses foram realizadas oficinas de trabalho nos Congressos de Epidemiologia e Saúde Coletiva da Abrasco nos anos de 2001 a 2003, quando foi possível congregar representação de outros centros de pesquisa e núcleos de saúde pública do País, disseminando os resultados do PPAS e iniciando um relacionamento em rede que pretendemos consolidar com o novo projeto colaborativo denominado de Ação Intersetorial para a Saúde.

No processo em curso avançou-se na compreensão de que, em contextos marcados por profundas desigualdades e iniqüidade sociais, as teorias e práticas da promoção da saúde mantêm estreito diálogo com a estratégia de desenvolvimento local integrado e sustentável (DLIS), na qual se incorporam os componentes de geração de trabalho e renda, desenvolvimento urbano e saneamento, educação e lazer numa perspectiva de gestão de políticas de forma intersetorial e participativa.

Considerando o grande interesse por parte dos governos federal, regionais e municipais na interlocução em rede entre experiências de gestão participativa, desenvolvimento integrado, municípios saudáveis com a incorporação dos pressupostos e propostas do campo da promoção da saúde, a CPHA/ENSP/Abrasco desenvolveram uma nova proposta, visando consolidar o setor saúde como parceiro-chave para a implementação de políticas de desenvolvimento local de forma a impactar positivamente a saúde e qualidade de vida da população.

O Projeto Ação Intersetorial para Saúde buscará, assim, apoiar os esforços do Brasil em promover desenvolvimento local e reduzir desigualdades sociais, estreitando a capacidade brasileira para a colaboração intersetorial e para implementação e avaliação de intervenções direcionadas aos determinantes sociais da saúde. Possibilitará, ainda, a consolidação de parcerias com diversos municípios e iniciativas através da sistematização, disseminação e produção de conhecimento sobre as estratégias de ação intersetorial e desenvolvimento local que vêm se desenvolvendo no campo da promoção da saúde no Brasil.

\section{Promoção da saúde, colaboração intersetorial e desenvolvimento local integrado e sustentável}

O desenvolvimento humano sustentável, conceito desenvolvido pelo Programa das Nações Unidas para o Desenvolvimento (PNUD), é uma das principais estratégias para a superação dos problemas sociais enfrentados pelas populações mais pobres. Tal prescrição está presente em inúmeros documentos recentes, gerados pelo consenso em eventos mundiais PNUD (1990 e seguintes); Banco Mundial (1990 e 1992); Nações Unidas (1992 e 1995). Mais recentemente, em outras áreas tem-se estabelecido propostas estritamente relacionadas ao conceito de desenvolvimento humano sustentável: na área ambiental, a Agenda 21, definida na Conferência das Nações Unidas sobre Meio Ambiente e Desenvolvimento - Rio/92; na área da saúde, a Promoção da Saúde (Canadá / OMS, 1986) e na área da habitação e dos assentamentos humanos, o direito à cidade e à moradia (HABITAT II, 1996).

No Brasil, a Constituição Federal de 1988 com rebatimento nas constituições estaduais e Leis Orgânicas Municipais - é pródiga em prescrições de direitos nestes campos. $\mathrm{O}$ artigo 196, por exemplo, estabelece com grande propriedade que "a saúde é um direito de todos e dever do Estado”, garantido mediante políticas sociais e econômicas que visem à redução do 
risco de doença e de outros agravos e ao acesso universal e igualitário às ações e serviços para sua promoção, proteção e recuperação. Estas premissas são as mesmas propostas anteriormente, em nível mundial, tanto na Declaração de Alma-Ata como na Carta de Otawa.

A Convenção de Ottawa determina que as condições fundamentais para a saúde são: paz, moradia, educação, alimentação, um ecossistema estável, recursos sustentáveis, justiça social e eqüidade. Além disso, a Convenção identifica cinco estratégias principais para a promoção da saúde: criar políticas públicas que visem à saúde, criar ambientes de apoio, fortalecer ações comunitárias, desenvolver habilidades individuais e reorientar os serviços de saúde.

Quando estes pré-requisitos e estratégias são considerados, torna-se evidente que a conquista de melhorias na saúde requer a interlocução com outros setores sociais, políticos e econômicos, numa ação intersetorial para a saúde (AIS). O Canadá tem experiência reconhecida neste campo. O Movimento de Comunidades para a Saúde, em Quebec, tem crescido de forma constante e apresenta papel importante ao levar a política pública em saúde a agir de forma mais sensível e integrada com a comunidade local. Também tem gerado importante reconhecimento internacional e, em 1996, as Comunidades para a Saúde do Quebec, com base na Universidade de Laval, foram designadas como Centro de Colaboração da OMS.

No Brasil, a estratégia de Desenvolvimento Local Integrado e Sustentável (DLIS) assume que qualquer plano de promoção de desenvolvimento social deve incluir políticas e ações capazes de englobar crescimento econômico e redução de desigualdades sociais e da pobreza. Sendo assim, o DLIS tem como conceito o desenvolvimento local como processo social em que o crescimento econômico é acompanhado por distribuição de riqueza e melhora na qualidade de vida, em uma determinada comunidade. Isto pode ser medido principalmente através do acesso da população aos recursos de saúde, educação, infra-estrutura assim como maior envolvimento das comunidades nas tomadas e decisão para alcance de melhores condições de vida.

O componente integrado baseia-se na idéia de que setores diferentes da vida comunitária e das políticas públicas devem definir e implementar políticas e ações conjuntas, baseadas nas prioridades da comunidade, segundo seus problemas, oportunidades e recursos.
Finalmente, a sustentabilidade deve ser considerada em suas várias dimensões, ambiental, política, econômica e cultural, garantindo que os resultados das ações serão contínuos e levarão a desenvolvimento futuro.

Para a consideração destes componentes na orientação das políticas de saúde, duas estratégias chaves têm sido destacadas: participação popular na definição das prioridades locais de desenvolvimento, através de processos de diagnóstico, planejamento e avaliação participativos; e colaboração intersetorial, através da coordenação do trabalho de diversos setores do governo e da comunidade, unidos para alcançar objetivos comuns.

Em suma, a promoção da saúde e o DLIS são guiados por valores comuns: uma visão abrangente da saúde e seus fatores determinantes, qualidade de vida, justiça e igualdade social, sustentabilidade e integração. Também têm estratégias de ação similares, baseadas em participação popular e colaboração intersetorial.

Há um relativo consenso de que na última década houve avanços no plano conceitural da promoção da saúde, particularmente no Brasil, pela coincidência de propósitos com a reforma sanitária e a consolidação do processo democrático. A ampliação do conceito de saúde como qualidade de vida e como direito humano fundamental, caracteriza-se como um imperativo ético de busca da autonomia dos sujeitos, da responsabilidade social e da eqüidade de oportunidades.

Neste sentido, entende-se como de fundamental importância para este novo projeto de cooperação técnica Brasil-Canadá estabelecer um amplo processo de produção de conhecimento, intercâmbio e fortalecimento das experiências no Brasil. $\mathrm{Na}$ realidade, apesar do grande número de iniciativas em desenvolvimento no País, são muito poucas as experiências que têm conseguido tirar do papel e levar à prática os conceitos de desenvolvimento humano sustentável, promoção da saúde e os demais conceitos convergentes mencionados acima, particularmente em territórios complexos como são as grandes cidades. Espera-se que a aplicação simultânea de tais conceitos e os métodos e práticas deles decorrentes possam trazer importante impacto social nas comunidades em que venham a ser aplicados. 


\section{Parcerias e intercâmbio de conhecimentos e práticas}

Considerando a existência no Brasil de inúmeras experiências em Promoção/DLIS e outras iniciativas de formação de redes, definiu-se inicialmente um número de seis experiências em curso como integrantes do Projeto e participantes nos fóruns de discussão e disseminação das experiências em um processo crescente de ampliação de parcerias.

Durante o processo de detalhamento do projeto foram realizadas oficinas de trabalho e visitas a municípios que estão desenvolvendo experiências de integração academia/gestão/comunidade, através de projetos intersetoriais e participativos voltados a locais de grande vulnerabilidade social, nas diversas regiões do país.

Desta forma, as experiências de Curitiba, Campinas, Itambé, Sobral e Goiás, além da experiência da ENSP em Manguinhos, descritas abaixo, respondem aos seguintes critérios:

- constituem iniciativas inovadoras de desenvolvimento local com protagonismo do setor saúde;

- apresentam forte vínculo com a comunidade local;

- desenvolvem colaboração academia/ serviço; e

- apresentam potencial de replicabilidade.

\section{Campinas (SP)}

A Universidade Estadual de Campinas (Unicamp), através de sua Pró-Reitoria de Extensão e Assuntos Comunitários desenvolve programas de promoção da saúde com ênfase na formação de agentes comunitários e na troca de experiências entre gestores e atores sociais dos municípios/comunidades da região noroeste no Estado de São Paulo, sudeste do Brasil. O Programa Comunidade Saudável e a iniciativa da Rede de Municípios Potencialmente Saudáveis, desenvolvidas em parceria com o Instituto de Pesquisas Especiais para a Sociedade (IPES) e a Organização Pan-Americana de Saúde (OPAS) envolvem, atualmente, 20 prefeituras da região, com população em torno de 1,5 milhão de habitantes. O Programa Comunidade Saudável, desde 1997, atua na formação de agentes comunitários e na construção de estratégias para o desenvolvimento local de cinco bairros da região dos Amarais em Campinas. Foram treinados, até o momento, 600 agentes comunitários. Em 2002, iniciou-se o processo de constituição da Rede de Municípios Potencialmente Saudáveis. Com uma metodologia de pesquisa-ação, foi realizado um levantamento das necessidades, problemas e estratégias para melhor otimização de recursos públicos visando à melhoria da qualidade de vida nos municípios. A partir desse processo, vem se promovendo reuniões entre gestores/profissionais de diversos setores e representantes das comunidades envolvidas para troca de experiências e reflexão sobre temáticas e propostas do campo da promoção da saúde.

\section{Curitiba (PR)}

A Pontifícia Universidade Católica (PUC) e a Secretaria Municipal de Saúde (SMS) de Curitiba, em parceria com outras cinco universidades, 23 empresas e 18 escolas de ensino fundamental lideram o Subprojeto Ambientes Saudáveis, como parte da estratégia de gestão pública municipal com base na intersetorialidade. Curitiba é a capital do Estado do Paraná, na região sul do Brasil, com população em torno de 1,6 milhão de habitantes. Apresenta um dos melhores índices de qualidade de vida do país. O Projeto Ambientes Saudáveis busca a integração entre as Secretarias de Saúde, de Esporte e Lazer, Cultura, Abastecimento, Ação Social e Educação no desenvolvimento de ações de promoção da saúde. Entre os anos de 2001 e 2003, em torno de 500 mil pessoas participaram dos 285 eventos realizados na cidade. Espaços públicos como parques e praças, e espaços comunitários como clubes, igrejas e associações de moradores, são transformados em áreas de democratização da educação em saúde, de estímulo à atividade física, de adoção de hábitos alimentares saudáveis, e atividades culturais e de lazer, educação ecológica, entre outros, criando oportunidades de participação que favorecem o "empoderamento" da população para hábitos mais saudáveis. Após alguns anos de experiência, foram escolhidos três ambientes/áreas na cidade, para dar suporte na promoção da saúde: universidades, empresas e escolas públicas de ensino fundamental, visando ao compromisso social destas instituições com a melhoria da qualidade de vida das pessoas, que passam a maior parte do tempo nestes espaços.

\section{Itambé (PE)}

O Núcleo de Saúde Coletiva e Desenvolvimento Social (NUSP) da Universidade Federal 
de Pernambuco (UFPE), em parceria com o governo do Estado e a prefeitura de Recife, com apoio da Agência de Cooperação Internacional do Japão (JICA), desenvolve o Projeto Cidades Saudáveis no município de Itambé. O município localiza-se na Zona da Mata, norte de Pernambuco, região nordeste do Brasil, com população de 34.920 habitantes, entre os quais 28.000 pessoas moram na zona urbana e apenas 6.000 na zona rural, com $97 \%$ da população envolvida na produção de cana-de-açúcar. Não há acesso a terra, ao trabalho, renda, lazer, saúde e/ou educação. O Projeto foi estruturado em 2001, seguindo metodologia pautada em cinco etapas: (1) a mobilização dos diferentes atores; (2) o autodiagnóstico, que é a percepção de cada um acerca da realidade; (3) o diagnóstico participativo, em que é aprofundada a percepção dos vários segmentos da realidade: saúde, educação, trabalho e renda, ambiente, política institucional, infra-estrutura. A realização desta etapa em 2002 contou com o apoio do Ministério da Saúde. O Projeto encontra-se atualmente na (4) etapa de desenho da agenda de prioridades para subsidiar a (5) etapa de mobilização de parceiros e recursos para colocar em prática o que o município definiu como proposta para mudar a realidade local. Um dos problemas sociais identificados é a questão da prostituição, como resultante da falta de opções de lazer para os jovens. Em convênio com o Unicef iniciaram o desenvolvimento de atividades culturais com uma comunidade em que havia um grande número de adolescentes grávidas, entre 11 e 15 anos de idade. O município conta hoje com um grupo de 200 “ativistas populares”, graças a um amplo trabalho de mobilização e um processo de nucleação que culminou com a realização da I Conferência Municipal de Saúde de Itambé. Em 2004, teve início o processo de expansão do Projeto Cidades Saudáveis para outros cinco municípios da Zona Agreste de Pernambuco através de convênio com a JICA.

\section{Sobral (CE)}

A Secretaria Municipal de Saúde de Sobral, através da Escola de Formação em Saúde da Família Visconde de Sabóia, desenvolve a experiência Estratégia Saúde da Família e a interface com as Políticas Públicas Municipais, em parceria com as demais Secretarias Municipais de Educação, Desenvolvimento Urbano e Rural, Esporte e Lazer, etc. Município com 165 mil habitantes, localizado na região norte do Cea- rá, Nordeste do Brasil, Sobral tem $86 \%$ de sua população localizada em área urbana. Tem como principal atividade econômica uma indústria de calçados que dá ocupação para $20 \mathrm{mil}$ pessoas. Como marca da gestão pública municipal de Sobral, a Estratégia Saúde da Família (ESF) vem sendo desenvolvida desde 1997 como parte da construção do Sistema Municipal de Saúde. A experiência vem se aprofundando com a realização do Curso de Especialização em nível de Residência em Saúde da Família; e com a crescente articulação das ações intersetoriais que destacam o espaço local (território), onde interagem a equipe de saúde da família, a comunidade e demais setores da administração pública. A cobertura da ESF é hoje próxima de 100\%. Existem 40 equipes implantadas no município, o que propiciou a melhoria de vários indicadores, em especial de mortalidade infantil. Os programas habitacionais, quando se dirigem a uma determinada área, atuam a partir da logística da ESF. Há todo um envolvimento e discussão dessas políticas com a equipe da ESF, congregando um conjunto de setores que estão organizados naquele território, a partir dos Conselhos Locais. Hoje, um dos pontos fortes em Sobral são os conselhos municipais, além do conselho municipal de saúde que está muito bem estruturado, há os de assistência social, de educação, de habitação, da criança e adolescente. Os conselhos de saúde e de assistência social são presididos por usuários.

\section{Goiânia (GO)}

A experiência de implementação de Processos Integrados de Gestão em Políticas Públicas no Estado de Goiás é coordenada pela Secretaria de Estado da Saúde (SES), em colaboração com demais Secretarias do Estado e da Universidade Estadual de Goiás. O projeto objetiva formular e validar processos metodológicos de integração e planejamento institucionais para adotá-los como estratégias gerenciais incorporadas à administração pública. O Estado de Goiás, composto por 246 municípios, está localizado no Planalto Central do Brasil. Sua população está estimada em 5,4 milhões de habitantes, sendo essencialmente jovem, onde 59\% possuem menos de 30 anos de idade. Sua capital, Goiânia, localiza-se a aproximadamente 200 km da capital federal, Brasília. Segundo estimativas do IBGE, atualmente, Goiânia conta com uma população de 1,4 milhão de habitantes, apresentando, na última década, um dos maio- 
res índices de crescimento populacional do País. A experiência iniciou em 2002 com a constituição de um Colegiado de Gestão dentro da SES visando à integração intrasetorial. Posteriormente, a convite da SES estabeleceu-se um processo de interlocução com as secretarias de Agricultura, Pecuária e Abastecimento; de Assuntos Institucionais, que lida com a questão da superintendência da mulher, infância e juventude; de Cidadania e Trabalho; de Ciência e Tecnologia, que é responsável pelas universidades; de Educação; do Meio Ambiente e Recursos Hídricos; de Habitação e Saneamento e a de Segurança Pública e Justiça. Durante o ano de 2003 foram realizadas as fases de capacitação, normatização e de regularização desse Grupo Intersetorial e, com o apoio da Unesco, foi desenvolvida uma metodologia de intervenção intersetorial para ser replicada nos municípios do Estado. A metodologia prevê a criação de grupo técnico-operacional local e realização de seminários com discussão das diversas formas de interação com as comunidades e formulação de projetos integrados. A fase atual é de definição de um município piloto para a implantação do projeto.

\section{Manguinhos (Rio de Janeiro RJ)}

A estratégia de Desenvolvimento Local Integrado e Sustentável em Manguinhos (DLIS) é uma iniciativa da ENSP/Fiocruz, em parceria com a Prefeitura da Cidade do Rio de Janeiro, empresas com sede na região e ONGs e lideranças locais participantes do Fórum Comunitário de Manguinhos. Manguinhos é um bairro da região norte do município do Rio de Janeiro, capital do Estado do Rio de Janeiro, na região sudeste do país. Concentra uma população em torno de 40 mil habitantes, em sua maioria moradores das 11 comunidades que circundam a Fiocruz. Considerado o quinto pior bairro em qualidade de vida, segundo o cálculo do IDH para todos os bairros da cidade do Rio de Janeiro, concentra problemas de infra-estrutura urbana, poluição do ar e dos rios, alto índice de analfabetismo e gravidez na adolescência, violência acentuada, característica das favelas e comunidades pobres da cidade. A partir de 1999, quando se iniciou o processo de mobilização de distintos atores para a elaboração de propostas nos campos da Saúde e Nutrição; Educação e Desenvolvimento Social; Geração de Renda; Urbanização; a estratégia DLISManguinhos vem agregando diversas iniciativas e projetos de intervenção, construção de conhecimento e formação/capacitação na área. Congrega atualmente, quatro grandes iniciativas: (1) a Reorientação da Atenção à Saúde do Centro de Saúde Escola Germano Sinval Farias da ENSP com ênfase na Estratégia Saúde da Família; (2) o Programa Universidade Aberta que desenvolve ações de promoção da saúde no campo da habitação e saneamento (3) o Laboratório Territorial de Manguinhos, voltado para a produção de conhecimento sobre riscos ambientais e tecnológicos e seus impactos na saúde junto com jovens estudantes do Ensino Médio da região; e (4) Grupo de ensino e pesquisa em avaliação de programas de promoção da saúde e desenvolvimento local, reunindo pesquisadores dos diversos departamentos da ENSP em colaboração com a Coordenação da Escola de Governo em Saúde.

As experiências selecionadas constituem, assim, programas complexos e inovadores de promoção da saúde, com reorientação da atenção, onde vários componentes estão presentes; ação pública intersetorial, participação comunitária, formação de agentes ou multiplicadores locais e parcerias com organizações sociais diversas. Estão sendo desenvolvidas por equipes multidisciplinares que abrangem tanto profissionais do setor saúde (acadêmicos e gestores), de outros setores das políticas sociais, assim como da própria comunidade organizada. Para participar diretamente das atividades deste projeto, foram definidas equipes locais de cada experiência que constituirão os núcleos irradiadores e disseminadores dos resultados do Projeto para demais atores e experiências locais e regionais.

O projeto de cooperação com o Canadá objetiva, portanto, potencializar as mudanças e resultados já alcançados nos programas de Promoção da Saúde selecionados, permitindo que tais resultados sejam discutidos, divulgados e disseminados, gerando conhecimento útil para todos os envolvidos e impactos de longo prazo no processo de redução das desigualdades sociais e de saúde em particular.

Artigo apresentado em 1ㅇ/7/2004

Aprovado em 7/7/2004

Versão final apresentada em 12/7/2004 